\title{
The Association of Fibroblast Growth Factor-23 with Mineral Factors (Ca, P, and Mg), Parathyroid Hormone, and 25-Hydroxyvitamin D in Hemodialysis Patients: A Multicenter Study
}

\author{
Farzanehsadat Minoo ${ }^{1,2}$, Elham Ramezanzade ${ }^{3,{ }^{*}}$, Mehrzad Mojarad ${ }^{1}$, Azam Alamdari ${ }^{1,2}$ and \\ Mohammad Taghi Najafi, ${ }^{1,2}$ \\ ${ }^{1}$ Nephrology Research Center, Tehran University of Medical Sciences, Tehran, Iran \\ ${ }^{2}$ Center of Excellence in Nephrology, Tehran University of Medical Sciences, Tehran, Iran \\ ${ }^{3}$ Organ Transplantation Research Center, School of Medicine, Guilan University of Medical Sciences, Rasht, Iran \\ Corresponding author: Organ Transplantation Research Center, Razi Hospital, School of Medicine, Guilan University of Medical Sciences, Rasht, Iran. Fax: +98-2166581568, \\ Email: rz.elham58@gmail.com
}

Received 2018 September 15; Accepted 2018 October 07.

\begin{abstract}
Background: Fibroblast growth factor-23 (FGF23) is a phosphaturic factor that is released from bone. A variety of bone diseases can occur in renal diseases.

Objectives: This study aimed to investigate the association of FGF23 with mineral factors, PTH, and 25-hydroxyvitamin D among hemodialysis patients.

Methods: This cross-sectional multicenter study was performed on 135 patients aged 18 years or over with end-stage renal disease treated with hemodialysis maintenance. FGF-23, phosphorus, Ca, Mg, PTH, 25-hydroxyvitamin D, Uric Acid, Na, and K were measured in each patient's fasting blood sample. We used univariate and multivariate linear regressions.

Results: The mean age of patients was $56.45 \pm 13.64$ years. The mean and median FGF23 concentration in patients were $855.07 \pm$ 43.33 and $762.6(\mathrm{IQR}=456.6-1430.3) \mathrm{pg} / \mathrm{mL}$, respectively. Different variables did not show any significant difference between the two sexes. After adjustment for age, sex, dialysis time, uric acid, Na, K, and kt/V, FGF23 had a linear association with 25-hydroxyvitamin D and every 10-unit ( $\mathrm{pg} / \mathrm{mL})$ increase in FGF23 was significantly associated with a $0.03 \mathrm{mg} / \mathrm{mL}$ increase in 25 -hydroxyvitamin $\mathrm{D}(\mathrm{P}=$ 0.04). In addition, other variables showed no significant association with FGF23.

Conclusions: According to the results, FGF23 had a linear association with vitamin D and an increase in FGF23 was significantly associated with an increase in vitamin D. In addition, there was no significant association between mineral factors and PTH, and FGF23.
\end{abstract}

Keywords: Fibroblast Growth Factor-23, Mineral Factors, Parathyroid Hormone, 25-hydroxyvitamin D, Hemodialysis Patients

\section{Background}

Fibroblast growth factor 23 (FGF23) is a hormone released from bone to plasma to increase phosphate excretion from the kidney. Besides, it is a predictor for the outcome in both acute and chronic illnesses (1-3). Increasing FGF23 levels have been shown in different stages of kidney diseases (1). Moreover, in adult patients with CKD, increased plasma FGF23 is associated with adverse outcomes. Bone disorders may occur very soon in CKD and there is a strong association between FGF23 and the risk of mortality mainly from cardiovascular disease, left ventricular hypertrophy, kidney disease progression, and vascular dysfunction in CKD (4-8).

Parathyroid hormone (PTH) is secreted to correct low levels of serum calcium. PTH increases serum Ca level through bone and kidney (9). PTH is commonly elevated in patients with CKD. The kidney is also the major site for producing 1,25 $(\mathrm{OH})_{2}$ D and 24,25 $(\mathrm{OH})_{2}$ D from $25(\mathrm{OH}) \mathrm{D}(10,11)$. High PTH often is found in people with vitamin D insufficiency. In contrast, FGF23 levels were elevated in those who were vitamin D replete (12). In addition, FGF23 is an important factor in regulating phosphorus $(\mathrm{P}), \mathrm{Ca}$, and vitamin D metabolism and it can lead to vascular calcifications (13). FGF23 that regulates vitamin D and $P$ homeostasis independent of the PTH shows potential molecular mechanisms of bone and mineral disorders in CKD (14).

The progressive derangement of mineral homeostasis is accompanied by CKD. Besides, there is an imbalance be- 
tween blood and tissue concentrations of Ca and P (15). Calcium and vitamin D can suppress PTH expression and parathyroid gland hyperplasia. It is believed that FGF23 and PTH mutually regulate each other in a negative feedback loop where PTH stimulates FGF23 production and FGF23, in turn, suppresses PTH synthesis (9). Therefore, FGF23, vitamin D, and PTH accompany each other and interact in the regulation of metabolism of $\mathrm{Ca}, \mathrm{P}$, and bone (13). Although variant derangements in calcium, phosphate, and vitamin D metabolism and complex bone diseases can occur in CKD, the association between different levels of these factors and the outcomes in hemodialysis patients is not completely understood (12).

Therefore, this study aimed to investigate the association of FGF23 with mineral factors (Ca, P, and Mg), PTH, and 25-hydroxyvitamin D among hemodialysis patients.

\section{Methods}

\subsection{Data Collection}

This cross-sectional multicenter study was performed on 135 patients aged 18 years or over with end-stage renal disease treated with hemodialysis maintenance from the hemodialysis center of Imam Khomeini Hospital in Tehran province and the hemodialysis center of Razi Hospital in Rasht city, Iran. Some necessary data regarding the patient's age, sex, and dialysis time were extracted from the records of the patients using a data collection form.

2.2. Mineral Factors ( $\mathrm{Ca}, \mathrm{P}$, and $\mathrm{Mg}$ ), Parathyroid Hormone (PTH), and 25-Hydroxyvitamin D Measurement in Patients

Phosphorus (P), calcium (Ca), magnesium (Mg), parathyroid hormone (PTH), and 25-hydroxyvitamin D were measured in each patient's blood sample before the beginning of dialysis using audit kits from Audit Company made in Ireland for Ca, P, and Mg; PTH was measured using chemiluminescent assay in IMMULITE2000 with Siemens kits from Siemens Company made in China; and 25-hydroxyvitamin D was determined using the ELFA (enzyme linked fluorescent assay) technique with Vidas kits from Vidas Company made in China.

\subsection{Biochemical Markers Measurement in Patients}

Uric acid, sodium ( $\mathrm{Na}$ ), and potassium (K) were measured using blood tests while fasting. In addition, kt/V was calculated in all patients.

\subsection{Measurement of Fibroblast Growth Factor 23}

FGF23 was measured by ELISA kits from BIOTECH company made in China before the beginning of dialysis sessions in $\mathrm{pg} / \mathrm{mL}$ unit.

\subsection{Statistical Analysis}

For a description of continuous data, first, the normality assumption was assessed using the KolmogorovSmirnov test. If data had a normal distribution, means and standard deviations were presented for them; otherwise, the medians and interquartile ranges were presented. For comparison of continues variables among males and females, we used a $t$-test for normal variables and MannWhitney test for non-normal distributed variables.

We used univariate and multivariate linear regressions to investigate the association between FGF23 and mineral factors (Ca, P, and Mg), parathyroid hormone, and 25 hydroxyvitamin $\mathrm{D}$. To better report and interpret the results, the FGF23 was divided by 10 . Hence, each unit change in FGF23 equals 10 pg/mL. Mineral factors (Ca, P, and Mg), PTH, and 25-hydroxyvitamin D were modeled in separate models against FGF23, dialysis time, age, sex, uric acid, Na, $\mathrm{K}$, and kt/V.

For $\mathrm{P}$ and $\mathrm{Mg}$, because the residuals of the models were normal, we did not use any outcome transformation. For PTH, because the residuals of the models were not normal, we did use log transformations. In addition, for $\mathrm{Ca}$ and 25-hydroxyvitamin $\mathrm{D}$, because the residuals of the models did not show normal distribution after various transformations, to get the correct P value and confidence interval, we used 300 bootstrap sampling. Data were analyzed by the Stata software (version 12). For all statistical tests, $\mathrm{P}<$ 0.05 was considered statistically significant.

\section{Results}

Using the Kolmogorov-Smirnov test, $\mathrm{P}, \mathrm{Mg}, \mathrm{Na}, \mathrm{K}$, and uric acid had normal distributions; hence, their means and standard deviations are presented. Other study variables had non-normal distribution and medians and interquartile ranges are presented for them.

Totally, 135 patients with end-stage renal disease were studied. The mean age of the patients was $56.45 \pm 13.64$ years (54.28 \pm 14.24 in males and $59.72 \pm 12.11$ in females). Sixty percent (81) of the patients were male (sex ratio: 1.5 male/female). The median dialysis time in patients was 7 $(\mathrm{IQR}=5-10)$ months. In addition, the mean and median FGF23 in patients were 855.07 \pm 43.33 and 762.6 $(\mathrm{IQR}=456.6$ - 1430.3) pg/mL, respectively. The distribution of $\mathrm{P}, \mathrm{Mg}, \mathrm{Na}$, $\mathrm{K}$, and uric acid among males and females was calculated by $t$-test and the distribution of dialysis time, FGF23, Ca, Vit. $\mathrm{D}, \mathrm{PTH}$, and kt/V among males and females was calculated by the Mann-Whitney test.

The mean age, dialysis time, kt/V, FGF23, Ca, P, PTH, 25hydroxyvitamin $\mathrm{D}$, and biochemical markers did not show any significant difference between the two sexes. The description of the study variables is presented in Table 1. 
There was a statistically significant association between FGF23 and dialysis time adjusted for sex and age. For every month increase in the duration of dialysis time, the concentration of FGF23 increased by $58.66 \mathrm{pg} / \mathrm{dL}(\mathrm{P}=0.001)$.

\subsection{Association of Mineral Factors ( $\mathrm{Ca}, \mathrm{P}$, and $\mathrm{Mg}$ ), Parathyroid Hormone, and 25-Hydroxyvitamin D Pressure with FGF23}

As Table 2 shows in univariate regression, there was no significant association between mineral factors (Ca, P, and $\mathrm{Mg}$ ), PTH, and 25-hydroxyvitamin D, and FGF23 (P > 0.05).

After adjustment for age, sex, dialysis time, uric acid, $\mathrm{Na}, \mathrm{K}$, and kt/V, FGF23 had a linear association with 25hydroxyvitamin D and every 10-unit $(\mathrm{pg} / \mathrm{mL})$ increase in FGF23 was significantly associated with a $0.03 \mathrm{mg} / \mathrm{mL}$ increase in 25-hydroxyvitamin $\mathrm{D}(\mathrm{P}=0.04)$. In addition, other variables did not show any significant association with FGF23 (Table 2).

\section{Discussion}

The aim of this study was to investigate the association between FGF23 and mineral factors (Ca, P, and Mg), parathyroid hormone, and 25-hydroxyvitamin $\mathrm{D}$ among patients with end-stage renal disease treated with hemodialysis maintenance.

Homeostasis of calcium and phosphate is maintained by active vitamin D in accompanying with FGF23 and PTH (16). In the present study, after adjustment for different variables, FGF23 had a linear association with vitamin D and every 10-unit (pg/mL) increase in FGF23 was significantly associated with a $0.03 \mathrm{mg} / \mathrm{mL}$ increase in vitamin D. Similarly, in a multicenter hemodialysis cohort in Taiwan, Chao et al. evaluated the relationship between 25 $(\mathrm{OH}) \mathrm{D}$ and 1,25 $(\mathrm{OH})_{2} \mathrm{D}$, vitamin D-binding protein, and FGF23. In multiple regression analyses, the serum FGF23 level had a strong association with total, free, and bioavailable $25(\mathrm{OH}) \mathrm{D}$ and total, free, and bioavailable $1,25(\mathrm{OH})_{2}$ D levels (17). In Mizuiri et al. study on 332 Japanese maintenance hemodialysis patients, the determinants of the serum FGF23 level in patients were age, serum Ca, P, PTH levels, the active vitamin D dose, and the GNRI (18). In addition, in Hsu et al. study, patients with higher FGF23 had higher $25(\mathrm{OH})$ vitamin $\mathrm{D}$, serum phosphorus, and age (19).

Previous studies have shown that FGF23 is adjusted by Ca, P, and PTH. FGF23 can suppress 1,25-dihydroxyvitamin D production and PTH secretion (16). On the other hand, the serum P level had a positive correlation with elevated FGF23 levels in patients with ESRD and the effects of PTH were variable (20). In addition, studies have shown that FGF23 could affect bone formation and mineralization, independently of its effect on phosphate regulation $(21,22)$.
Nevertheless, in our study, there was no significant association between mineral factors (Ca, P, and $\mathrm{Mg}$ ) and PTH, and FGF23. Contrary to our results, Sridharan et al. showed a strong association between different levels of FGF23 and the bone formation marker PINP. They also showed FGF23 was up-regulated following intermittent PTH levels (23). In contrast, in a study, the exogenous PTH administration reduced circulating FGF23 concentrations (24).

The reason why we did not find a relationship between calcium and phosphorus, and parathyroid hormone can be that despite a better understanding of FGF23 biology in systemic regulation of P turnover (25), factors inducing its skeletal expression have not been yet fully documented. 1,25-dihydroxyvitamin D, P, Ca, leptin, iron, secreted klotho, acidosis, and PTH are the factors currently known to induce FGF23 production (9). Perhaps, another reason we could not relate these factors is that our patients were not identical regarding the flux of dialysis filters. According to the results of Kendrick study, in hemodialysis patients, FGF23 levels are even more than 1000 times higher than in the normal population (26). Early increase in the level of FGF23 in CKD is an adaptive mechanism to prevent phosphate overload (27). In another study, over a period of 12 months, high-flux hemodialysis was associated with stable FGF23 levels whereas the low-flux hemodialysis group showed an increase in FGF23 (28).

In Wetmore et al. study, increased baseline log FGF23 levels had a significant correlation with putative alterations in gland mass as estimated by significantly shallower slopes of the iCa/PTH suppression curves. Besides, they reported that FGF23 levels decrease during dialysis, but the decrease does not appear to be associated with the changes in PTH or decrements of P during the hemodialysis procedure (29). Therefore, another reason may be that we did not measure the dynamic level of FGF 23 hormone during dialysis, as the Wetmore et al. study may have an effect on its slope.

As a result, the mean and median FGF23 were 855.07 \pm 43.33 and $762.6(\mathrm{IQR}=456.6$ - 1430.3 $) \mathrm{pg} / \mathrm{mL}$, respectively. In addition, the mean age of the patients was $56.45 \pm 13.64$ years and the median dialysis time in patients was seven months. In Jean et al. study, the mean serum FGF23 level was $7060 \pm 1350 \mathrm{RU} / \mathrm{mL}$ and linear regressions showed a significant correlation between log FGF23 and age. In addition, the mean age was $66.6 \pm 14$ years (30). In Negishi et al. study, the serum FGF23 level in dialysis patients was $1171 \pm 553 \mathrm{pg} / \mathrm{mL}$. The level of FGF23 was significantly higher in hemodialysis patients than in healthy volunteers (1171 vs. $48 \mathrm{pg} / \mathrm{mL}$ ) (31). Therefore, all studies indicated a higher level of FGF23 in renal disease and CKD patients. Similarly, Chao et al. reported that the mean age of patients was 66.4 \pm 13.9 years (17). 


\begin{tabular}{|c|c|c|c|c|}
\hline Variables & Male $(\mathbf{n}=\mathbf{8 1})$ & Female $(n=54)$ & Total $(\mathbf{n}=135)$ & PValue \\
\hline & $\operatorname{Median}(\text { Q1 - Q3) })^{\mathrm{a}}$ & Median (Q1 - Q3) & Median (Q1 - Q3) & \\
\hline Dialysis time (mo) & $7(5-10)$ & $8(4-11)$ & $7(5-10)$ & $0.64^{\mathrm{b}}$ \\
\hline FGF23 (pg/mL) & $701.5(423.3-1430.3)$ & $822.5(475-1424)$ & $762.6(456.6-1430.3)$ & $0.45^{\mathrm{b}}$ \\
\hline $\mathrm{Ca}(\mathrm{mg} / \mathrm{mL})$ & $8.7(8.3-9.1)$ & $9(8.4-9.5)$ & $8.8(8.3-9.3)$ & $0.12^{\mathrm{b}}$ \\
\hline Vit. D (mg/mL) & $37(25.3-53.9)$ & $31(19-57.6)$ & $37(21-57)$ & $0.37^{\mathrm{b}}$ \\
\hline PTH (ng/mL) & $325(173-613)$ & $337(202-550)$ & $325(191-575)$ & $0.86^{\mathrm{b}}$ \\
\hline \multirow[t]{2}{*}{$\mathbf{k t} / \mathbf{V}$} & $1.2(1.14-1.24)$ & $1.19(1.18-1.22)$ & $1.2(1.15-1.23)$ & $0.65^{\mathrm{b}}$ \\
\hline & Mean (SD) & Mean (SD) & Mean (SD) & \\
\hline $\mathbf{P}(\mathbf{m g} / \mathbf{m L})$ & $5.38(1.3)$ & $5.16(1.13)$ & $5.29(1.23)$ & $0.29^{c}$ \\
\hline $\operatorname{Mg}(\mathbf{m g} / \mathbf{m L})$ & $2.16(0.26)$ & $2.26(0.30)$ & $2.20(0.28)$ & $0.03^{c}$ \\
\hline $\mathrm{Na}(\mathrm{meq} / \mathrm{L})$ & $139.22(3.91)$ & $139.05(4.63)$ & $139.15(4.2)$ & $0.82^{\mathrm{c}}$ \\
\hline $\mathbf{K}(\mathbf{m e q} / \mathbf{L})$ & $4.58(0.63)$ & $4.77(0.84)$ & $4.66(0.72)$ & $0.15^{\mathrm{c}}$ \\
\hline Uric acid (mg/dL) & $6.5(1.33)$ & $6.49(1.32)$ & $6.50(1.32)$ & $0.94^{\mathrm{c}}$ \\
\hline
\end{tabular}

${ }^{\mathrm{a}} \mathrm{Q} 1$ - Q3 = Interquartile range.

${ }^{\mathrm{b}}$ Based on Mann-Whitney test.

${ }^{\mathrm{c}}$ Based on $t$-test.

Table 2. The Univariate and Multivariate Association of Mineral Factors (Ca, P, and Mg), PTH, and 25-Hydroxyvitamin D with FGF23 Among Hemodialysis Patients ${ }^{\mathrm{a}}$, b

\begin{tabular}{|c|c|c|c|c|c|c|c|c|}
\hline \multirow{2}{*}{ Dependent Variables } & \multicolumn{3}{|c|}{ Univariate } & \multicolumn{5}{|c|}{ Multivariate } \\
\hline & $\beta$ & SE & PValue & $\beta$ & SE & 95\% CI & P Value & $\mathbf{R}^{2}$ \\
\hline $\mathrm{Ca}(\mathrm{mg} / \mathrm{mL})$ & -0.0004 & 0.001 & 0.81 & -0.0002 & 0.002 & $-0.005-0.005$ & 0.93 & 0.02 \\
\hline $\mathbf{P}(\mathbf{m g} / \mathbf{m L})$ & 0.002 & 0.002 & 0.32 & 0.003 & 0.002 & $-0.001-0.007$ & 0.13 & 0.08 \\
\hline $\operatorname{Mg}(\mathbf{m g} / \mathbf{m L})$ & -0.0002 & 0.0004 & 0.59 & -0.0001 & -0.0005 & $-0.001-0.0009$ & 0.84 & 0.11 \\
\hline PTH (ng/mL) & 0.001 & 0.001 & 0.25 & 0.001 & 0.001 & $-0.001-0.004$ & 0.48 & 0.08 \\
\hline Vit. $D(\mathbf{m g} / \mathbf{m L})$ & 0.04 & 0.03 & 0.19 & 0.06 & 0.03 & $0.002-0.13$ & 0.04 & 0.16 \\
\hline
\end{tabular}

${ }^{a} \beta$, regression coefficient; $C I$, confidence interval; $\mathrm{R}^{2}$, coefficient of determination; $\mathrm{SE}$, standard error

${ }^{\mathrm{b}}$ Based on linear regression and adjusted for: age, sex, dialysis time, uric acid, Na, Kand kt/V.

\subsection{Conclusion}

According to the results, FGF23 had a linear association with vitamin D and an increase in FGF23 was significantly associated with an increase in vitamin D. In addition, there was no significant association between mineral factors and PTH, and FGF23.

\section{Acknowledgments}

We would like to express our thanks to all the hemodialysis patients and the staff of Hemodialysis Center of Imam Khomeini Hospital in Tehran and Razi Hospital in Rasht, Iran, as well as to all individuals helping us in completing this research project.

\section{Footnotes}

Authors' Contribution: Study concept and design: Farzanehsadat Minoo and Elham Ramezanzadeh; analysis and interpretation of data: Mehrzad Mojarad; drafting of the manuscript: Azam Alamdari and Mohammadtaghi Najafi; critical revision of the manuscript for important intellectual content: Farzanehsadat Minoo, Elham Ramezanzadeh, Mehrzad Mojarad, Azam Alamdari and Mohammadtaghi Najafi; statistical analysis: Farzanehsadat Minoo and Elham Ramezanzadeh.

Conflict of Interests: All authors declare that they have no conflict of interest.

Ethical Considerations: All procedures performed in the study involving data extraction from existing information were in accordance with the ethical standards of the Tehran University of Medical Sciences Research Committee 
and with the 1964 Helsinki Declaration and its later amendments or comparable ethical standards.

Funding/Support: This study was supported by Tehran University of Medical Sciences (grant number 940214629538). The funding agency did not play any role in the planning, conduct, and reporting of the manuscript or in the decision to submit it for publication.

\section{References}

1. Coresh J, Turin TC, Matsushita K, Sang Y, Ballew SH, Appel LJ, et al. Decline in estimated glomerular filtration rate and subsequent risk of end-stage renal disease and mortality. JAMA. 2014;311(24):2518-31. doi: 10.1001/jama.2014.6634. [PubMed: 24892770]. [PubMed Central: PMC4172342].

2. Schnedl C, Fahrleitner-Pammer A, Pietschmann P, Amrein K. FGF23 in acute and chronic illness. Dis Markers. 2015;2015:358086. doi: 10.1155/2015/358086. [PubMed: 26491212]. [PubMed Central: PMC4600945].

3. Scialla JJ, Xie H, Rahman M, Anderson AH, Isakova T, Ojo A, et al. Fibroblast growth factor-23 and cardiovascular events in CKD. J Am Soc Nephrol. 2014;25(2):349-60. doi: 10.1681/ASN.2013050465. [PubMed: 24158986]. [PubMed Central: PMC3904568].

4. Park M, Shlipak MG, Katz R, Agarwal S, Ix JH, Hsu CY, et al. Subclinical cardiac abnormalities and kidney function decline: The multiethnic study of atherosclerosis. Clin J Am Soc Nephrol. 2012;7(7):113744. doi: 10.2215/CJN.01230212. [PubMed: 22580783]. [PubMed Central: PMC3386670].

5. Lundberg S, Qureshi AR, Olivecrona S, Gunnarsson I, Jacobson SH, Larsson TE. FGF23, albuminuria, and disease progression in patients with chronic IgA nephropathy. Clin J Am Soc Nephrol. 2012;7(5):72734. doi: 10.2215/CJN.10331011. [PubMed: 22383747]. [PubMed Central: PMC3338280]

6. Isakova T, Xie H, Yang W, Xie D, Anderson AH, Scialla J, et al. Fibroblast growth factor 23 and risks of mortality and end-stage renal disease in patients with chronic kidney disease. JAMA. 2011;305(23):24329. doi: 10.1001/jama.2011.826. [PubMed: 21673295]. [PubMed Central: PMC3124770].

7. Faul C, Amaral AP, Oskouei B, Hu MC, Sloan A, Isakova T, et al. FGF23 induces left ventricular hypertrophy. J Clin Invest. 2011;121(11):4393408. doi: 10.1172/JCI46122. [PubMed: 21985788]. [PubMed Central: PMC3204831].

8. Seiler S, Reichart B, Roth D, Seibert E, Fliser D, Heine GH. FGF-23 and future cardiovascular events in patients with chronic kidney disease before initiation of dialysis treatment. Nephrol Dial Transplant. 2010;25(12):3983-9. doi: 10.1093/ndt/gfq309. [PubMed: 20525642].

9. Lanske B, Razzaque MS. Molecular interactions of FGF23 and PTH in phosphate regulation. Kidney Int. 2014;86(6):1072-4. doi: 10.1038/ki.2014.316. [PubMed: 25427080]. [PubMed Central: PMC4246422].

10. Lawson DE, Fraser DR, Kodicek E, Morris HR, Williams DH. Identification of 1,25-dihydroxycholecalciferol, a new kidney hormone controlling calcium metabolism. Nature.1971;230(5291):228-30. [PubMed: 4323313].

11. Holick MF, Schnoes HK, DeLuca HF, Gray RW, Boyle IT, Suda T. Isolation and identification of 24,25-dihydroxycholecalciferol, a metabolite of vitamin D made in the kidney. Biochemistry. 1972;11(23):4251-5. [PubMed: 4342902].

12. Taal MW, Thurston V, McIntyre NJ, Fluck RJ, McIntyre CW. The impact of vitamin D status on the relative increase in fibroblast growth factor 23 and parathyroid hormone in chronic kidney disease. Kidney Int. 2014;86(2):407-13. doi: 10.1038/ki.2013.537. [PubMed: 24429404].
13. Shardlow A, McIntyre NJ, Fluck RJ, McIntyre CW, Taal MW. Associations of fibroblast growth factor 23, vitamin D and parathyroid hormone with 5-year outcomes in a prospective primary care cohort of people with chronic kidney disease stage 3. BMJ Open. 2017;7(8). e016528. doi: 10.1136/bmjopen-2017-016528. [PubMed: 28838895]. [PubMed Central: PMC5629682].

14. Stubbs J, Liu S, Quarles LD. Role of fibroblast growth factor 23 in phosphate homeostasis and pathogenesis of disordered mineral metabolism in chronic kidney disease. Semin Dial. 2007;20(4):302-8. doi: 10.1111/j.1525-139X.2007.00308.x. [PubMed: 17635819].

15. Nitta K, Nagano N, Tsuchiya K. Fibroblast growth factor 23/klotho axis in chronic kidney disease. Nephron Clin Pract. 2014;128(1-2):1-10. doi: 10.1159/000365787. [PubMed: 25402964].

16. Shimada T, Hasegawa H, Yamazaki Y, Muto T, Hino R, Takeuchi Y, et al. FGF-23 is a potent regulator of vitamin D metabolism and phosphate homeostasis. J Bone Miner Res. 2004;19(3):429-35. doi: 10.1359/JBMR.0301264. [PubMed: 15040831].

17. Chao CT, Lee SY, Yen CJ, Chiang CK, Huang JW, Hung KY. Serum free 1,25-dihydroxy-vitamin D is more closely associated with fibroblast growth factor 23 than other vitamin $\mathrm{D}$ forms in chronic dialysis patients. Clin Chim Acta. 2015;439:122-7. doi: 10.1016/j.cca.2014.10.022. [PubMed: 25451947].

18. Mizuiri S, Nishizawa Y, Yamashita K, Ono K, Oda M, Usui K, et al. Lower serum fibroblast growth factor-23 levels may suggest malnutrition in maintenance haemodialysis patients. Nephrology (Carlton). 2014;19(9):568-73. doi: 10.1111/nep.12290. [PubMed: 24947687].

19. Hsu JJ, Katz R, Ix JH, de Boer IH, Kestenbaum B, Shlipak MG. Association of fibroblast growth factor-23 with arterial stiffness in the Multi-Ethnic Study of Atherosclerosis. Nephrol Dial Transplant. 2014;29(11):2099-105. doi: 10.1093/ndt/gfu101. [PubMed: 24782533]. [PubMed Central: PMC4209876].

20. Weber TJ, Liu S, Indridason OS, Quarles LD. Serum FGF23 levels in normal and disordered phosphorus homeostasis. J Bone Miner Res. 2003;18(7):1227-34. doi: 10.1359/jbmr.2003.18.7.1227. [PubMed: 12854832].

21. Shen J, Fu S, Song Y. Relationship of fibroblast growth factor 23 (FGF23) serum levels with low bone mass in postmenopausal women. J Cell Biochem. 2017;118(12):4454-9. doi: 10.1002/jcb.26101. [PubMed: 28464278].

22. Yoshiko Y, Wang H, Minamizaki T, Ijuin C, Yamamoto R, Suemune $S$, et al. Mineralized tissue cells are a principal source of FGF23. Bone. 2007;40(6):1565-73. doi: 10.1016/j.bone.2007.01.017. [PubMed: 17350357].

23. Sridharan M, Cheung J, Moore AE, Frost ML, Fraser WD, Fogelman I, et al. Circulating fibroblast growth factor-23 increases following intermittent parathyroid hormone (1-34) in postmenopausal osteoporosis: Association with biomarker of bone formation. Calcif Tissue Int. 2010;87(5):398-405. doi: 10.1007/s00223-010-9414-8. [PubMed: 20838781].

24. Samadfam R, Richard C, Nguyen-Yamamoto L, Bolivar I, Goltzman D. Bone formation regulates circulating concentrations of fibroblast growth factor 23. Endocrinology. 2009;150(11):4835-45. doi: 10.1210/en.2009-0472. [PubMed: 19819968].

25. Razzaque MS. The FGF23-Klotho axis: Endocrine regulation of phosphate homeostasis. Nat Rev Endocrinol. 2009;5(11):611-9. doi: 10.1038/nrendo.2009.196. [PubMed: 19844248]. [PubMed Central: PMC3107967].

26. Kendrick J, Cheung AK, Kaufman JS, Greene T, Roberts WL, Smits G, et al. FGF-23 associates with death, cardiovascular events, and initiation of chronic dialysis. J Am Soc Nephrol. 2011;22(10):1913-22. doi: 10.1681/ASN.2010121224. [PubMed: 21903574]. [PubMed Central: PMC3187186].

27. Prie D, Urena Torres P, Friedlander G. Latest findings in phosphate homeostasis. Kidney Int. 2009;75(9):882-9. doi: 10.1038/ki.2008.643. [PubMed: 19190675]. 
28. Schneider A, Schneider MP, Krieter DH, Genser B, Scharnagl H, Stojakovic T, et al. Effect of high-flux dialysis on circulating FGF-23 levels in end-stage renal disease patients: Results from a randomized trial. PLoS One. 2015;10(5). e0128079. doi: 10.1371/journal.pone.0128079. [PubMed: 26024521]. [PubMed Central: PMC4449206].

29. Wetmore JB, Santos PW, Mahnken JD, Krebill R, Menard R, Gutta H, et al. Elevated FGF23 levels are associated with impaired calciummediated suppression of PTH in ESRD. J Clin Endocrinol Metab. 2011;96(1):E57-64. doi: 10.1210/jc.2010-1277. [PubMed: 20943782]. [PubMed Central: PMC3038477].
30. Jean G, Terrat JC, Vanel T, Hurot JM, Lorriaux C, Mayor B, et al. High levels of serum fibroblast growth factor (FGF)-23 are associated with increased mortality in long haemodialysis patients. Nephrol Dial Transplant.2009;24(9):2792-6. doi:10.1093/ndt/gfp191. [PubMed: 19395730].

31. Negishi K, Kobayashi M, Ochiai I, Yamazaki Y, Hasegawa H, Yamashita $\mathrm{T}$, et al. Association between fibroblast growth factor 23 and left ventricular hypertrophy in maintenance hemodialysis patients. Comparison with B-type natriuretic peptide and cardiac troponin T. Circ J. 2010;74(12):2734-40. [PubMed: 21041973]. 